\title{
PhD Nursing Students' Perceptions Towards Clinical Informatics Course
}

\author{
Jialin LIU ${ }^{\mathrm{a}, \mathrm{b}, 1}$, Siri LIU' ${ }^{\mathrm{c}}$, Mei FU' ${ }^{\mathrm{d}}$ and Jinbo FANG ${ }^{\mathrm{e}}$ \\ ${ }^{a}$ Department of Medical Informatics, West China Hospital, Sichuan University, China \\ ${ }^{\mathrm{b}}$ Department of Medical Informatics, West China School of Medicine, \\ Sichuan University, Chengdu, China \\ ${ }^{c}$ Department of Biomedical Informatics, University of Utah, Salt Lake City, Utah, USA \\ ${ }^{d}$ Outpatient Department, West China Hospital, Sichuan University, Chengdu, China \\ ${ }^{\mathrm{e}}$ West China School of Nursig, West China Hospital, Sichuan University, China
}

\begin{abstract}
This paper aims to investigate $\mathrm{PhD}$ nursing students' perceptions regarding a clinical informatics course. Open-ended questionnaires and reviews were used to explore the students' perception of the course. A total of $84.62 \%$ (11/13) students responded to the survey. Only four respondents had an understanding of clinical informatics and others did not. All the respondents considered clinical informatics to be a very important and useful course for $\mathrm{PhD}$ nursing students.
\end{abstract}

Keywords. Clinical informatics, course, nursing student, perception

\section{Introduction}

With the development of the digital hospital, clinical informatics is embedded in nursing education programs in a variety of ways, including curriculum integration and standalone course $[1,2]$. Students' perceptions are critical for course design and evaluation. It will help to refine the course and adjust content to students' needs. This pilot study collected and analyzed $\mathrm{PhD}$ nursing students' perceptions of clinical informatics course at West China School of Medicine, Sichuan University, China. This school is one of the most famous medical schools and a national model of higher medical education in China.

\section{Methods}

We developed an open-ended questions survey in consultation with medical educators and informatics experts. The completion of the questionnaires was voluntary. We surveyed all $\mathrm{PhD}$ nursing students (13) joining the course in 2019. The questionnaire includes the following three questions: 1) Talk about your understanding of clinical informatics. 2) What are you hoping to learn from the course? 3) Why do you take clinical informatics as an elective course? The answers to all questionnaires were entered

\footnotetext{
${ }^{1}$ Corresponding Author, Jialin Liu, Department of Medical Informatics, West China Hospital, Sichuan University, Chengdu, Sichuan, China; E-mail: dlj18@163.com.
} 
into Microsoft office excel 2007, and qualitative content analysis was performed by reviewers.

\section{Results}

Of the 13 potential participants, 11 respondents replied to all the questionnaires. The survey response rate was $84.62 \%(11 / 13)$. Table 1 shows the characteristics of $\mathrm{PhD}$ nursing students. All the respondents answered that clinical informatics is a very useful course for $\mathrm{PhD}$ nursing students, so we have elected clinical informatics. Four respondents $(4 / 11,36.4 \%)$ have a better understanding of clinical informatics. Seven respondents $(7 / 11,33.6 \%)$ have an incomplete understanding of clinical informatics. They consider clinical informatics to be equivalent to medical information retrieval.

All the respondents $(11 / 11,100 \%)$ hope to learn the application of information technology in clinical practices and described what they want to learn through the course. Four themes emerged from the 32 data items mentioned by the interviewees. The most common themes were: information technology in nursing $(9 / 11,81.8 \%)$, data mining $(5 / 11,45.5 \%)$, database $(4 / 11,36.4 \%)$, and medical big data $(4 / 11,36.4 \%)$.

Table 1. Characteristics of the interviewed PhD nursing students.

\begin{tabular}{clc}
\hline Demographics & Number & Mean (SD) \\
\hline Age & $25-41$ years & $30.83 \pm 5.15$ \\
Gender & & \\
Female & 10 & \\
Male & 1 & $5.65 \pm 5.84$ \\
Length of tenure & $0-19$ years & \\
$5<$ & 5 & \\
$5-10$ & 4 & \\
$>10$ & 2 & \\
\hline
\end{tabular}

\section{Conclusion}

All the respondents thought clinical informatics is a very useful course for PhD nursing students. They all hope to learn more about clinical informatics. The students' perceptions are also helpful to design and refine the clinical informatics course. Further research will refine the course according to students' perceptions, and evaluate the refined outcome.

\section{References}

[1] Guerra D. Teaching clinical informatics in a concept-based flipped classroom. Nurse Educ 44 (2019),129-131.

[2] Sorenson J, Campell L. Curricular path to value: Integrating an academic electronic health record. J Nurs Educ 55 (2016), 716-719. 